\title{
Adaptação brasileira da Escala de Atitudes Disfuncionais (DAS)
}

\author{
Mara Rúbia de Camargo Alves Orsini ${ }^{1}$ \\ Marcelo Tavares \\ Bartholomeu Tôrres Tróccoli
}

\begin{abstract}
Resumo
A Escala de Atitudes Disfuncionais (DAS) avalia crenças e pressuposições que podem criar alguma vulnerabilidade cognitiva para depressão. Este estudo examinou a consistência, a estrutura interna da DAS-A e DAS-B e suas correlações com o Inventário Beck de Depressão (BDI). Esses instrumentos foram aplicados a uma amostra de duzentos e setenta e três estudantes universitários, com idades entre 19 e 25 anos (68,8\%), solteiros $(85,7 \%)$, mulheres $(62,6 \%)$, e cursando os primeiros dois anos $(63,7 \%)$ de faculdade. Os resultados revelaram índices aceitáveis de confiabilidade e uma boa estrutura fatorial das duas formas da DAS, demonstrando que elas podem ser consideradas como equivalentes. Todavia, as correlações entre ambas as formas e o BDI sugeriram uma não-especificidade da escala com sintomas de depressão. Novos procedimentos que melhorem as propriedades da escala poderão habilitar seu uso não somente em futuras pesquisas em psicopatologia, mas também possibilitarão o uso clínico da escala DAS.

Palavras-chave: Atitudes disfuncionais; Escala; Propriedades psicométricas; Depressão; Vulnerabilidade cognitiva.
\end{abstract}

\section{Brazilian version of the Dysfunctional Attitude Scale (DAS)}

\begin{abstract}
The Dysfunctional Attitude Scale (DAS) evaluate beliefs and assumptions that may create some cognitive vulnerability to depression. This study examined the consistency; internal structure of the DAS-A and DAS-B and their correlations with the Beck Depression Inventory (BDI). These questionnaires were applied to a sample of two hundred seventy three university students, with ages between 19 and 25 years old $(68,8 \%)$, single $(85,7 \%)$, female $(62,6 \%)$, and studying in their first two years $(63,7 \%)$ at the university. The results revealed acceptable indices of reliability and a good factorial structure of the two forms of DAS showing that they could be considered as equivalents. However, the correlations between both forms and with the BDI suggested a non specificity of the scale with symptoms of depression. New improvement of the properties of the scale would enable not only further research on psychopathology but could also allow the clinical use of the DAS scale.
\end{abstract}

Keywords: Dysfunctional attitudes; Scale; Psychometric properties; Depression; Cognitive vulnerability.

\section{Introdução}

Os estudos - no campo da psicopatologia - de Beck (1963, 1964, 1971); Beck, Ward, Mendelson, Mock e Erbaugh (1961) e de Ward, Beck e Rascoe (1961) introduziram um postulado teórico cognitivo para a depressão, atribuindo um papel central às cognições na caracterização do quadro geral dessa patologia. Conforme o referencial teórico de Beck, as pessoas fazem uso de regras e premissas para ajustarem suas próprias vidas. Ao lidarem com outras pessoas, rotulam, interpretam e avaliam a si mesmas, aos outros e às situações de acordo com essas regras. Quando estas são estruturadas de maneira rígida, irrealística, podem conduzir a um desajustamento que se torna fonte de dificuldades adaptativas e conseqüente sofrimento para a pessoa. Essas cognições são denominadas de regras e atitudes disfuncionais.
Beck preocupou-se com o desenvolvimento e validação de instrumentos que propiciassem maior consistência e adequação diagnóstica. Um instrumento inicial, que reflete tal preocupação, é o Inventário Beck de Depressão - BDI (Beck e colaboradores, 1961), traduzido e adaptado para o Brasil por Cunha (2001), que se centra em assertivas que expressam, além de sintomas físicos, cognições típicas de pacientes deprimidos.

O BDI (Beck e colaboradores, 1961) constitui um meio bastante confiável para avaliação clínica da depressão, porém não possibilita a avaliação dos pressupostos teóricos de Beck sobre o processamento cognitivo, característico da depressão. Diante disso, Weissman (1979) procurou construir um instrumento que identificasse as pressuposições subjacentes ao processamento cognitivo dessa patologia. Essas cognições constituiriam um fator de vulnerabilidade para depres-

\footnotetext{
${ }^{1}$ Endereço para correspondência:

Universidade Católica de Goiás - UCG, Departamento de Psicologia

Av. Universitária, 1440 - Setor Universitário - Caixa Postal 86 - 74605-010 - Goiânia-GO

E-mail: maraipc@yahoo.com.br, mararubia.mr@gmail.com
} 
são, que é o que a Escala de Atitudes Disfuncionais DAS - (Weissman, 1979) objetiva avaliar.

A Escala de Atitudes Disfuncionais (DAS) foi desenvolvida num estudo inicial de Weissman e Beck (1978) e validada por Weissman (1979). Originalmente, a escala foi desenvolvida a partir de uma lista de itens baseados no referencial teórico de Beck e que, após serem aplicados a residentes de psiquiatria, que também serviram de juízes, resultou nos 100 itens originais da escala. Com o objetivo de maior brevidade, sem perda da confiabilidade, a escala foi fatorializada. Os resultados indicaram que 10 fatores foram extraídos dos 100 itens da DAS. Todavia, Weissman decidiu, nesse estudo inicial da escala, tratar o construto de distorções cognitivas de uma maneira unidimensional e, por isso, Weissman concentrou-se no primeiro fator extraído que identifica a dimensão mais geral e contribui com a máxima variância comum entre todos os itens. Os resultados da análise fatorial foram, então, usados para construir duas formas paralelas da DAS, com 40 itens cada: DAS-A e DAS-B (Weissman, 1979).

Essa escala foi avaliada em diversos estudos sobre suas propriedades psicométricas, tanto em amostras de estudantes, como em amostras clínicas (Beck, Brown, Steer \& Weissman, 1991; Calhoon, 1996; Cane, Olinger, Gotlib \& Kuiper, 1986; Dyck, 1992; Nelson, Stern \& Cicchetti, 1992; Oliver \& Baumgart, 1985; Parker, Bradshaw \& Blignault, 1984; Power, Katz McGuffin, Duggan, Lam \& Beck, 1994;), inclusive em outros contextos culturais (Chioqueta \& Stiles, 2004; Sahin \& Sahin, 1992) e é referida na literatura como a medida de vulnerabilidade cognitiva à depressão mais comumente utilizada (Brown, Hammen, Craske \& Wickens, 1995).

No estudo de Power e colaboradores (1994), foi utilizada uma amostra composta por 277 sujeitos entre estudantes universitários (Open University), pacientes deprimidos (Maudsley Hospital) e parentes desses pacientes - que completaram um conjunto de questionários enviados pelo correio: DAS-A e B e uma medida geral de sintomas. A análise da consistência interna, nesse trabalho, foi calculada através do alfa de Cronbach e evidenciou bons coeficientes, tanto para a DAS-A quanto para a DAS-B: 0,898 e 0,860 , respectivamente (Power e colaboradores, 1994).

No estudo apresentado por Oliver e Baumgart (1985), o coeficiente alfa foi verificado na avaliação da consistência interna da DAS, apresentando os seguintes resultados, numa amostra de 275 sujeitos, para a versão original da escala (DAS-100), para a DAS-A e para a DAS-B. O coeficiente alfa para as três formas foi de 0,90, 0,85 e 0,81, para a DAS-100, DAS-A e DAS-B, respectivamente. As confiabilidades de formas paralelas para a DAS-A e B foram todas satisfatoriamente altas: $0,83,0,86$ e $0,81 \quad(\phi<0,0001)$, para a amostra total $(n=275)$, homens $(n=105)$ e mulheres $(n=170)$, respectivamente (Oliver \& Baumgart, 1985).

Já no estudo de Nelson e colaboradores (1992), são apresentados resultados sobre a consistência interna da DAS-100 (versão original da escala, com 100 itens), bem como da DAS-A e DAS-B (versão estatisticamente reduzida), numa amostra clínica de pacientes deprimi$\operatorname{dos}(n=72)$ e não-deprimidos $(n=1)$.

A consistência interna das escalas, nesse estudo, foi calculada através do Alfa de Cronbach, obtendo-se os seguintes coeficientes: para a DAS-100: 0,96 (amostra total), 0,97 (deprimidos) e 0,95 para os não-deprimidos. Para a análise das duas formas A e B, os resultados foram: DAS-A 0,89 (não-deprimidos), 0,94 (para a amostra total e para os deprimidos); e para a forma B da escala os resultados obtidos foram: 0,88 (nãodeprimidos), 0,93 (deprimidos) e 0,92 para a amostra total. Tais resultados indicam boa estabilidade interna tanto para a DAS-100, quanto para as duas formas reduzidas da escala - DAS-A e DAS-B (Nelson e colaboradores, 1992). Vale ressaltar que esses valores mais altos para o coeficiente alfa, encontrados nesse estudo, são esperados devido à utilização de amostra clínica, o que aumenta a variabilidade da amostra.

$\mathrm{Na}$ versão Norueguesa para a forma A da escala, Chioqueta e Stiles (2004) avaliaram 344 jovens recrutas militares, 41 sujeitos saudáveis, que serviram de controle e 142 pacientes psiquiátricos ambulatoriais. A análise da DAS-A revelou um alfa de Cronbach de 0,85, indicando uma confiabilidade satisfatória. Evidência para a validade de construto foi obtida pela correlação entre a DAS (forma A) e o Inventário Beck de Depressão - BDI $(r=0,47)$ e entre a DAS e o Automatic Thoughts Questionnaire $(r=0,47)$. Finalmente, a Escala de Atitudes Disfuncionais, nessa amostra, discriminou significativamente entre pacientes clinicamente deprimidos, pacientes psiquiátricos não-deprimidos e sujeitos controle saudáveis. Os resultados, de acordo com Chioqueta e Stiles (2004), mostraram que a versão Norueguesa da DAS possui propriedades psicométricas satisfatórias, sugerindo que esse instrumento é apropriado para uso como medida cognitiva em um contexto cultural norueguês.

A equivalência das duas versões da escala também foi objeto de verificação em diversos estudos, principalmente através de análise fatorial (Beck e colaboradores, 1991; Calhoon, 1996; Oliver \& Baumgart, 1985; Parker e colaboradores, 1984; Power e colaboradores, 1994).

$\mathrm{Na}$ análise fatorial, conduzida por Power e colaboradores (1994), a solução de três fatores para os 
dados da DAS-A $(n=144)$ e para a DAS-B $(n=133)$, foi a que se revelou mais interpretável. A carga mínima (somente em um fator), para que cada item fosse selecionado, foi de 0,40 . O primeiro fator contou com 22,5\% para a variância total e seus itens estão relacionados à realização e medo de fracasso e foi nomeado de realização. O segundo fator contou com 6,2\% da variância e os itens são concernentes a questões relativas à dependência $\mathrm{e}$ aprovação, e foi rotulado de dependência. $\mathrm{O}$ terceiro fator contribuiu com $5,4 \%$ da variância e apresentou uma certa dificuldade para interpretação, comparados aos fatores 1 e 2. Seus itens parecem incluir questões tanto de dependência como de autocontrole, e foi nomeado autocontrole/dependência. Portanto, a variância total explicada pelos 3 fatores soma 34,1\%.

Os resultados da análise fatorial para a DAS-B foram: $17,7 \%$ da variância total devidos ao fator 1, que foi rotulado de realização, apesar de que seus itens pareciam conter várias questões sobre dependência, comparados aos itens do fator realização equivalente para a DAS-A. O segundo fator respondeu por $10,1 \%$ da variância total e contém itens que estão relacionados a autocontrole e parecem mais bem definidos do que o fator equivalente mais próximo para a DAS-A (ou seja, fator 3). O terceiro fator contribuiu para $6,1 \%$ da variância total e contém itens que parecem relacionar-se com questões sobre dependência. Contudo, a presença do item: "a pessoa deve procurar por uma solução prática para os problemas mais do que por uma solução perfeita $(0,66)$ " complicou um pouco a interpretação desse fator (Power e colaboradores, 1994).

Ainda com relação à estrutura interna das forma estatisticamente reduzidas da escala (DAS-A e DAS-B), alguns estudos como o de Parker e colaboradores (1984) e de Oliver e Baumgart (1985) encontraram uma configuração fatorial de 4 fatores e já apresentaram maior dificuldade no estabelecimento de equivalência entre as formas A e B da DAS.

A Escala de Atitudes Disfuncionais (DAS), apesar de ainda apresentar algumas contradições na literatura, principalmente no que diz respeito à equivalência ou não de suas formas reduzidas (DAS-A e DAS-B), tem alcançado, por outro lado, índices razoáveis de fidedignidade, bem como bons critérios em diversos processos de validação, mostrando ser útil e válida a ampliação de seu estudo, para fins de pesquisa e diagnóstico.

O uso de um instrumento como a DAS pode representar um ganho diagnóstico e prognóstico, visto que todo paciente ao buscar ajuda traz consigo toda uma bagagem de crenças, atitudes e expectativas que têm influenciado, de certa forma, o curso de sua problemática e as possibilidades de um resultado favorável. O objetivo do presente estudo é avaliar as propriedades psicométricas das duas formas reduzidas da Escala de Atitudes Disfuncionais (DAS-A e DAS-B) de Weissman (1979), sua consistência e sua estrutura interna e verificar se existe uma relação entre atitudes disfuncionais e sintomas depressivos, isto é, avaliar a especificidade das duas versões da escala para a depressão clínica.

\section{Método}

Sujeitos

Um total de 273 estudantes, de uma universidade privada de porte médio, foi avaliado neste estudo. Do total, $62,6 \%$ eram do sexo feminino ( $\mathrm{n}=$ $171)$ e $37,4 \%$ eram do sexo masculino $(n=102)$. Desse total, $68,8 \%$ tinham idade entre 19 e 25 anos. A maior parte $(85,7 \%)$ era composta por solteiros. Da amostra $58,6 \%$ trabalhavam, dos quais $51,3 \%$ tinham renda pessoal inferior a $\mathrm{R} \$ 600,00$. A renda familiar de $65,4 \%$ estava entre $R \$ 1.201,00$ e $R \$ 6.000,00$. A maior parte dos sujeitos cursava o $1 .^{\circ}$ e o $2 .^{\circ}$ anos $(36 \%$ e $27,7 \%$, respectivamente). Os cursos freqüentados pelos sujeitos avaliados se distribuíram entre as áreas de Humanas (39,8\%), Exatas (37,1\%) e Biológicas $(23,1 \%)$ e $96 \%$ da amostra não havia concluído nenhum outro curso superior.

Com relação às características clínicas da amostra, verificou-se que $75,1 \%$ dos sujeitos pesquisados não se consultaram nem uma vez com um psicólogo ou psiquiatra. Ademais, 86,1\% nunca estiveram em tratamento psicoterapêutico e 95,6\% nunca receberam medicação psiquiátrica. Consultados como se sentiam nos últimos dias, 85,3\% se colocaram na porção superior da Escala de Bem-Estar (Tavares, 1995).

\section{Instrumentos}

Escala de Atitudes Disfuncionais - DAS (Weissman, 1979). O instrumento possui duas formas (DAS-A/DAS-B), 40 itens em cada versão e uma escala de pontuação do tipo Likert com sete alternativas de resposta. Cada item expressa uma crença ou atitude, por exemplo: "meu valor enquanto pessoa depende muito do que os outros pensam de mim", sendo acompanhados de sete alternativas de respostas, onde $1=$ concordo totalmente e $7=$ discordo totalmente. As propriedades psicométricas da DAS foram avaliadas em diversos estudos. Por exemplo, Oliver e Baumgart (1985) encontraram coeficientes alfa de 0,85 e 0,81 para as formas A e B da DAS, respectivamente. Bem como uma confiabilidade teste-reteste (seis semanas) de 0,73 , numa amostra de 275 adultos. Já o estudo de Cane e 
colaboradores (1986), realizado somente com a forma A da escala em uma amostra de 664 universitários, identificou um coeficiente alfa de 0,87 . Para o presente estudo, duas traduções das versões A e B da Escala de Atitudes Disfuncionais (DAS) foram produzidas: uma por um psicólogo clínico bilíngüe e outra por uma aluna do último ano do curso de psicologia, fluente em inglês. A comparação dessas duas traduções funcionou como um primeiro teste semântico. Uma segunda validação semântica foi feita, submetendo-se os itens duvidosos ao julgamento de outros dois professores especialistas em Psicologia Clínica, com fluência no idioma.

Inventário Beck de Depressão - BDI (Beck e colaboradores, 1961): O BDI é um instrumento de autorelato, contendo 21 itens que avaliam a presença e a severidade de manifestações do humor, bem como manifestações cognitivas, motivacionais, psicomotoras e vegetativas, típicas da depressão. Para cada item, são apresentadas quatro alternativas de respostas, constituídas por afirmativas que expressam a intensidade ou severidade do sintoma ( 0 = "Não me sinto triste"; 1 = "Sinto-me triste"; 2 = "Estou triste o tempo todo"; $3=$ "Não consigo sair dessa"; 4 = "Estou tão triste ou infeliz que não agüento mais"). Em sua adaptação para o Brasil, Cunha (2001) encontrou um coeficiente alfa de 0,82 para o BDI, em uma amostra de 1.746 estudantes universitários.

Escala de Bem-estar: Esta escala foi elaborada por Tavares (1995) para avaliar o bem-estar recente do indivíduo. Uma única pergunta solicita ao respondente que descreva a maneira como tem se sentido nos últimos dias utilizando uma escala onde 1= "excelente, nunca estive melhor"; 2 = "muito bem"; 3 = "bem"; 4 = "mais para melhor do que para pior"; 5 = "mais para pior do que para melhor"; $6=$ "ruim"; 7 = "muito ruim" e 8= "péssimo, nunca estive pior".

Questionário Demográfico / Condição Clínica. Para a coleta dos dados demográficos foi utilizado um questionário com perguntas genéricas, objetivando a caracterização da amostra, no qual foram incluídas também três questões de cunho clínico, relevantes para este estudo ("Você já se consultou, pelo menos uma vez na sua vida, com um psicólogo ou psiquiatra?"; "Você alguma vez já fez psicoterapia?"; "Você alguma vez já recebeu medicação psiquiátrica?’”).

\section{Procedimentos}

Os instrumentos foram aplicados em sala de aula, com a permissão prévia do professor, sendo a participação dos alunos voluntária. Os sujeitos receberam um envelope contendo um questionário para a coleta dos dados demográficos e informações sobre as características clínicas da amostra e os testes DAS-A, DAS-B e BDI. A seqüência de aplicação foi feita de forma contrabalançada, de modo que metade da amostra recebeu os instrumentos e os respondeu na seqüência: DAS-A / DAS-B / BDI (daqui por diante rotulada de seqüência ou ordem de aplicação $A / B)$ e a outra metade respondeu na ordem: DAS-B / DAS-A / BDI (seqüência ou ordem de aplicação B/A).

\section{Resultados}

Em uma primeira análise dos resultados obtidos com as escalas DAS, foram consideradas como variáveis independentes as formas da DAS (DAS-A ou DAS-B), o sexo dos participantes e as ordens nas quais as duas formas da DAS foram respondidas (Ver Tabela 1/ Anexo A). Os resultados da análise de variância $2 \times 2 \times$ 2 realizada nos dados da Tabela 1 revelaram a presença de duas interações significativas. $\mathrm{Na}$ primeira, as variáveis Formas da DAS e Ordem de Aplicacão interagiram significativamente $[\mathrm{F}=4,38 ; p<0,04]$, isto é, a média da forma $\mathrm{A}$ diferiu da média da forma $\mathrm{B}$ apenas quando a forma A foi aplicada antes da forma B; quando a ordem de aplicação foi invertida, as médias das duas formas não diferiram entre si. A segunda interação significativa ocorreu entre as Formas da $D A S$ e o Gênero [F $=6,25 ; p<0,01]$. Neste último caso, os homens apresentam uma média menor de atitudes disfuncionais apenas quando avaliados com a forma $\mathrm{A}$.

Tabela 1 - Médias e desvios padrão das atitudes disfuncionais nas escalas a e b, de acordo com a ordem de aplicação e o gênero dos participantes

\begin{tabular}{ccccc}
\hline & \multicolumn{2}{c}{ Forma A } & \multicolumn{2}{c}{ Forma B } \\
\hline & Masculino & Feminino & Masculino & Feminino \\
Ordem A/B & 129,4 & 126,2 & 128,8 & 118,2 \\
& $(\mathrm{dp}=19,60)$ & $(\mathrm{dp}=20,11)$ & $(\mathrm{dp}=20,47)$ & $(\mathrm{dp}=22,42)$ \\
Ordem B/A & 117,7 & 122,4 & 119,9 & 121,0 \\
& $(\mathrm{dp}=24,43)$ & $(\mathrm{dp}=25,30)$ & $(\mathrm{dp}=23,57)$ & $(\mathrm{dp}=24,65)$ \\
\hline
\end{tabular}


Quando os participantes foram avaliados com a forma $\mathrm{B}$ da DAS, os homens apresentaram, em comparação com as mulheres, uma média claramente maior de atitudes disfuncionais. Estes resultados sugerem baixa correspondência entre as duas formas uma vez que tanto o gênero quanto a ordem de aplicação afetam diferencialmente seus resultados.

As correlações entre as formas da DAS de acordo com a ordem de aplicação (0,67 para seqüência
A/B e 0,72 para a seqüência B/A) foram razoáveis, porém um pouco discrepantes, já que correspondem a diferentes percentuais de variância em comum (45\% vs $52 \%)$. A correlação média geral $(0,70 ; p<0,01)$ entre as duas formas da DAS indica, portanto, uma correspondência moderada entre elas (49\% de variância compartilhada). Tais resultados aproximam, no entanto, do obtido por outros pesquisadores que consideram as formas A e B como formas paralelas (e.g., Oliver \&

Tabela 2 - Itens da DAS-A, agrupados de acordo com a solução de três fatores, suas respectivas cargas fatoriais e alfa de Cronbach $(n=264)$.

\begin{tabular}{|c|c|}
\hline Item & $\begin{array}{c}\text { Carga } \\
\text { no } \\
\text { fator }\end{array}$ \\
\hline \multicolumn{2}{|l|}{$\begin{array}{l}\text { Fator 1: baixa auto-estima indutora } \\
\qquad \text { alfa }=0,71\end{array}$} \\
\hline Se eu não for tão bem sucedido quanto as outras pessoas, isso significa que sou um ser humano inferior. & 0,70 \\
\hline Se falho no meu trabalho, então sou um fracasso enquanto pessoa. & 0,62 \\
\hline Pessoas que têm boas idéias são mais dignas que aquelas que não têm. & 0,53 \\
\hline Se uma pessoa pede ajuda, é um sinal de fraqueza. & 0,50 \\
\hline Se alguém discorda de mim, provavelmente isto indica que esta pessoa não gosta de mim. & 0,44 \\
\hline Pareço alguém inferior quando faço uma pergunta. & 0,37 \\
\hline Se as pessoas souberem como você realmente é, elas terão menos consideração por você. & 0,36 \\
\hline Não sou nada se quem eu amo não me ama. & 0,35 \\
\hline Se eu não for sempre bem sucedido as pessoas não me respeitarão. & 0,33 \\
\hline
\end{tabular}

\section{Fator 2: baixa auto-estima induzida por objeto esquemático} alfa $=0,57$

É horrível ser desaprovado pelas pessoas com as quais nos importamos.

Para eu ser uma pessoa de valor, devo ser excelente em pelo menos um aspecto importante. 0,47

Para ser uma pessoa boa, moral e digna, devo ajudar a todos que precisam. 0,45

Estar isolado das outras pessoas acaba levando à infelicidade. 0,45

Não posso ser feliz a não ser que a maioria das pessoas que conheço me admirem. 0,42

Se você não tem em quem se apoiar, você está fadado a ser uma pessoa triste. 0,42

Cometer erros é bom porque posso aprender a partir deles. $-0,40$

Se eu não exigir o mais alto padrão de mim mesmo, estou fadado a ser uma pessoa de segunda categoria. 0,35

Se eu cometo um erro costumo ficar chateado.

\section{Fator 3: atitudes mantenedoras da depressão}

$$
\text { alfa }=0,64
$$

Não posso ser feliz a não ser que a maioria das pessoas que conheço me admire.

Posso ser feliz, mesmo se eu perder muitas das coisas boas da vida.

O meu valor enquanto pessoa depende muito do que os outros pensam de mim.

Minha felicidade depende mais dos outros do que de mim.

Uma pessoa pode ter prazer em uma atividade independentemente do resultado final.

É possível conquistar o respeito das pessoas sem ser especialmente talentoso em nada.

É possível uma pessoa ser repreendida e não se chatear.

Eu não preciso da aprovação alheia para conseguir ser feliz.

Posso encontrar a felicidade sem ser amado por outra pessoa.

Cometer erros é bom porque posso aprender a partir deles.

É difícil ser feliz a não ser que se seja uma pessoa de boa aparência, inteligente, rica e criativa. 
Baumgart, 1985; Power e colaboradores, 1994).

Com relação à consistência interna, as correlações item-total apresentaram valores de $-0,0086$ a 0,5832 (DAS-A) e 0,0824 a 0,5236 (DAS-B). Esses valores estão abaixo dos resultados dos demais estudos da literatura revisada, o que possivelmente reflete a presença de alguns itens inadequados. Mas os valores do coeficiente alfa $(0,81$ para a forma DAS-A e 0,80 para a forma DAS-B) não diferem muito dos reportados na literatura.

Os resultados das correlações entre atitudes disfuncionais e sintomas depressivos $(r=0,22$ para os testes DAS-A/BDI e $r=0,21$ para DAS-B/BDI), embora significativas $(p<0,01)$ refletem claramente uma validade convergente inadequada entre a DAS e o BDI, visto que este índice não chega a $10 \%$ de variância em

Tabela 3 - Itens da DAS-B agrupados de acordo com a solução de três fatores, suas respectivas cargas fatoriais e alfa de Cronbach $(n=264)$.

Item

Carga

no

Fator

\section{Fator 1: baixa auto-estima induzida por autoprescrição irrealizável}

$$
\text { alfa }=0,77
$$

Eu deveria ser capaz de resolver meus problemas rapidamente e sem muito esforço. 0,59

Eu deveria ser capaz de agradar a todos.

Uma pessoa deve ser bem sucedida em todos os seus empreendimentos.

Devo ser feliz o tempo todo.

Uma pessoa deve ser capaz de controlar tudo o que lhe acontece.

Devo sempre ter controle total sobre os meus sentimentos. 0,52

Devo tentar impressionar as pessoas se quero que gostem de mim. 0,42

Tenho de impressionar os novos conhecidos com meu charme, inteligência ou senso de humor, ou então 0,40 não gostarão de mim.

As pessoas devem preparar-se para o pior, senão elas ficarão decepcionadas. 0,36

É horrível quando as pessoas com quem eu me importo não gostam de mim. 0,35

Se eu colocar o interesse das pessoas antes do meu, elas deverão me ajudar quando eu quiser que elas 0,34

façam algo por mim.

Se alguém é indiferente comigo, isso significa que não gosta de mim.

\section{Fator 2: baixa auto-estima indutora$$
\text { alfa }=0,75
$$

Se uma pessoa não é bem sucedida, então sua vida não tem sentido.

A não ser que eu seja um sucesso, minha vida terá sido um desperdício.

As pessoas podem se importar comigo, mesmo se conhecerem minhas fraquezas. 0,53

As pessoas o rejeitarão se conhecerem suas fraquezas.

0,46

Se digo uma bobagem, isso significa que sou uma pessoa boba.

As pessoas que têm a marca do sucesso (boa aparência, fama e fortuna) certamente serão mais felizes que 0,44 as que não têm.

Se a pessoa que eu amo não me ama, isso significa que não sou passível de ser amado.

Devo tentar impressionar as pessoas se quero que gostem de mim.

Sempre que me arrisco ou faço uma tentativa, só estou procurando confusão. 0,32

Para uma pessoa é vergonhoso demonstrar sua fraqueza.

\section{Fator 3: atitudes mantenedoras da depressão}

$$
\text { alfa }=0,64
$$

Eu não preciso da aprovação dos outros para ser feliz.

É possível eu me curtir mesmo quando os outros não gostam de mim.

Uma pessoa não precisa ser benquista para ser feliz.

Meu valor enquanto pessoa depende muito daquilo que os outros pensam de mim. 0,44

Se as pessoas não me acham atraente isto não me aborrece necessariamente. 0,34

Devo tentar impressionar as pessoas se quero que gostem de mim. 
comum entre as duas escalas o que, naturalmente, nos limita com relação à possibilidade de interpretação de uma vulnerabilidade específica para a depressão. O tamanho dessas correlações, portanto, sugere uma nãoespecificidade das escalas com sintomas de depressão, conforme avaliados pelo BDI.

Análises Fatorais exploratórias foram realizadas com as duas formas da DAS, a análise dos componentes principais (PC) foi seguida pela rotação varimax, verificando-se a existência de três fatores explicando $24,7 \%$ da variância para a forma $\mathrm{A}$ e $26,1 \%$ para a forma B. A carga mínima adotada para que o item fosse selecionado foi de 0,30 .

Para a DAS-A (Tabela 2/Anexo B), o primeiro fator (baixa auto-estima indutora, alfa $=0,71,9$ itens) contribuiu com $13,7 \%$ para a variância total e seus itens estão relacionados a sentimentos de baixa auto-estima que levariam à seleção dos aspectos negativos da avaliação sobre si mesmo, que o indivíduo acredita ser feita pelo outro, como no item "Se eu não for tão bem sucedido quanto as outras pessoas, isso significa que sou um ser humano inferior".

O fator 2 da DAS-B (baixa auto-estima indutora, alfa $=0,75 ; 10$ itens) contribuiu com $7,6 \% \mathrm{da}$ variância e o conteúdo de seus itens também sugerem tal como o fator 1 da DAS-A -, uma baixa auto-estima associada, vinculada à leitura negativa do julgamento que ele acredita que o outro fará dele, como expressa, por exemplo, o conteúdo dos itens tais como "se uma pessoa não é bem sucedida, então sua vida não tem sentido" ou "as pessoas o rejeitarão se conhecerem suas fraquezas".

O fator 3 para a DAS-B contribuiu com 4,7\% da variância total (alfa $=0,64 ; 6$ itens) e seu conteúdo também se assemelha ao conteúdo do fator 3 da DASA que é composto fundamentalmente pelos itens reversos ou adaptativos e refletem assim atitudes patológicas de dependência, próprias da depressão, conforme elaborado anteriormente. Este fator foi também rotulado de Atitudes Mantenedoras da Depressão, como exemplificado no item "eu não preciso da aprovação dos outros para ser feliz".

Resumindo, a proximidade conceitual entre as formas A e B reflete um consenso entre as duas formas da escala DAS com relação a seus construtos subjacentes, no que diz respeito a esta amostra. Outros índices, no entanto, limitam um pouco a equivalência entre as formas.

\section{Discussão}

O interesse inicial pela Escala de Atitudes Disfuncionais (DAS) justifica-se em razão da Psico-USF, v. 11, n. 1, p. 25-33, jan./jun. 2006 potencialidade do instrumento, como um método mais objetivo e simples de avaliação diagnóstica - no sentido de possibilitar a avaliação das pressuposições subjacentes à leitura que o paciente faz do mundo, de si mesmo e do futuro. Um instrumento dessa natureza, que apresente bons índices psicométricos, constitui-se em um meio prático e impessoal para acessar as assunções ou inferências que o indivíduo faz acerca de sua realidade interna e externa.

No entanto, a escala DAS ainda apresenta, de acordo com a literatura revisada, algumas controvérsias, a respeito de suas propriedades psicométricas. Vários estudos relatam uma concordância conceitual entre as formas A e B da escala (Parker e colaboradores, 1984), bem como bons índices de confiabilidade (Dobson \& Breiter, 1983; Oliver \& Baumgart, 1985; Cane e colaboradores, 1986; Nelson e colaboradores, 1992; Chioqueta \& Stiles, 2004), mas não há um consenso geral com relação a tópicos específicos tais como a equivalência ou não da estrutura interna das duas formas reduzidas da escala (Oliver \& Baumgart, 1985), ou dos coeficientes de correlação entre ambas (Power e colaboradores, 1994).

Neste estudo procurou-se investigar se versões em português da escala DAS apresentariam características psicométricas adequadas. Os resultados revelaram índices de consistência interna da escala que se mantiveram dentro de limites aceitáveis, não se distanciando muito dos resultados obtidos por outros estudos. O mesmo ocorreu quanto à estrutura interna da escala. A escala parece apresentar uma estrutura conceitual estável, pois a análise de sua estrutura interna revelou construtos (fatores) condizentes com estudos prévios (Power e colaboradores, 1994), significativos e compatíveis entre si. Esta estabilidade conceitual da escala, através de diversos estudos - envolvendo diferentes características de método - indica uma adequação e uma direção correta no processo da validação das dimensões inerentes à escala.

A interpretação conceitual da estrutura fatorial da Escala de Atitudes Disfuncionais, também a recomenda como fonte possível para a avaliação das crenças adotadas pelo paciente e assume relevância como fonte de pesquisa, principalmente, pelo fato de que ela pode apresentar implicações técnicas, ligadas a questões diagnósticas e prognósticas. Uma boa ilustração disso pode ser encontrada no trabalho de Peselow, Robins, Block, Barouche e Fieve (1990), onde o papel dos padrões mal adaptativos de pensamentos é avaliado em relação ao prognóstico de pacientes deprimidos. Observou-se, nesse estudo, que quanto mais alta a classificação inicial de atitudes disfuncionais, conforme avaliada pela DAS, mais pobre a resposta ao 
tratamento, isto é, mais reservado o prognóstico do paciente. Estes dados são condizentes com os de outros estudos, como o de Eaves e Rush (1984) que também encontraram alguma consistência com relação a um escore mais alto na DAS predizer um prognóstico menos favorável. Isto pode representar implicações técnicas importantes para a prática clínica, no sentido de possibilitar a seleção de intervenções terapêuticas mais apropriadas a esse diagnóstico prévio, propiciado pela escala.

O presente estudo constitui uma avaliação inicial da escala, em amostra brasileira e demanda, naturalmente, novos estudos que poderão ampliar os dados obtidos nessa primeira investigação. Esta, apesar de apresentar algum consenso com os dados relatados na literatura, indica que outros procedimentos metodológicos se fazem importantes, para que a escala seja utilizada com mais propriedade e confiabilidade.

Dentre esses procedimentos, um próximo passo seria a condução de análises estatísticas e semânticas dos itens da escala, visto que os resultados obtidos ainda sugerem a existência de itens inadequados. Isso exige que, em estudos futuros, os itens que não se mostraram bons no presente trabalho, ou por uma fraca correlação Item-Total, ou por não terem apresentado cargas fatoriais substantivas, sejam reformulados de maneira mais apropriada a nosso contexto ou, em último caso, sejam retirados da escala. Talvez, uma outra questão de importância fundamental esteja ligada a questão da qualidade dos itens: diferenças culturais, levantadas por estudos tais como o de Sahin e Sahin (1992), onde são pontuadas algumas divergências com relação à validade e discriminação dos itens reversos ou adaptativos da escala, em estudos transculturais.

Outro aspecto importante a ser investigado, em estudos futuros, diz respeito à especificidade da escala para avaliar vulnerabilidade para depressão, visto que nossos resultados, na correlação entre a DAS e o BDI, apesar de apresentarem índices positivos, não foram, por sua vez, relevantes. Isto parece sugerir uma nãoespecificidade para esta patologia, isto é, a escala parece avaliar mais dimensões do que aquelas específicas de vulnerabilidade para sintomas depressivos (o que também já foi indicado em outros estudos, como o de Dyck, 1992). Para tanto, em estudos futuros, seria interessante verificar-se a correlação da escala com outras medidas de sintomas, além da depressão.

Por último, não se deve esquecer que a amostra deste primeiro estudo foi composta por sujeitos universitários, o que, de certa forma, limita e impõe a necessidade de que outros resultados sejam obtidos em outras amostras, outros contextos e outras vivências, principalmente aquelas advindas de amostras clínicas.

\section{Referências}

Beck, A. T. (1963). Thinking and depression: I. Idiosyncratic content and cognitive distortions. Archives of General Psychiatry, 9, 324-333.

Beck, A. T. (1964). Thinking and depression: II. Theory and therapy. Archives of General Psychiatry, 10, 561-571.

Beck, A. T. (1971). Cognition, affect, and psychopathology. Archives of General Psychiatry, 24, 495-500.

Beck, A. T., Brown, G., Steer, R. A. \& Weissman, A. N. (1991). Factor analysis of the Dysfunctional Attitude Scale in a clinical population. Psychological Assessment: A Journal of Consulting and Clinical Psychology, 3, 478483.

Beck, A. T., Ward, C. H., Mendelson, M., Mock, J. \& Erbaugh, J. (1961). An inventory for measuring depression. Archives of General Psychiatry, 4, 561-571.

Brown, G. P., Hammen, C. L., Craske, M. G. \& Wickens, T. D. (1995). Dimensions of dysfunctional attitudes as vulnerabilities to depressive symptoms. Journal of Abnormal Psychology, 104, 431-435.

Calhoon, S. K. (1996). Confirmatory factor analysis of the Dysfunctional Attitude Scale in a student sample. Cognitive Therapy and Research, 20, 81-91.

Cane, D. B., Olinger, L. J., Gotlib, I. H. \& Kuiper, N. A. (1986). Factor structure of the Dysfunctional Attitude Scale in a student population. Journal of Clinical Psychology, 42, 307-309.

Chioqueta, A. P. \& Stiles T. C. (2004). Psychometric properties of the Norwegian version of the Dysfunctional Attitude Scale (Form A). Cognitive Behavaviour Therapy, 33(2), 83-86.

Cunha, J. A. (2001). Manual da versão em português das Escalas Beck. São Paulo: Casa do Psicólogo.

Dobson, K. S. \& Breiter, H. J. (1983). Cognitive assessment of depression: reliability and validity of three measures. Journal of Abnormal Psychology, 92, 107109.

Dyck, M. J. (1992). Subscales of the Dysfunctional Attitude Scale. British Journal of Clinical Psychology, 31, 333-335.

Eaves, G. \& Rush, A. J. (1984). Cognitive patterns in symptomatic and remitted unipolar major depression. Journal of Abnormal Psychology, 93, 31-40. 
Nelson, L. D., Stern, S. L. \& Cicchetti, D. V. (1992). The Dysfunctional Attitude Scale: how well can it measure depressive thinking? Journal of Psychopathology and Behavioral Assessment, 14, 217-223.

Oliver, J. M. \& Baumgart, E. P. (1985). The Dysfunctional Attitude Scale: psychometric properties and relation to depression in an unselected adult population. Cognitive Therapy and Research, 9, 161167.

Parker, G., Bradshaw, G. \& Blignault, I. (1984). Dysfunctional attitudes: measurement, significant constructs and links with depression. Acta Psychiatrica Scandinavia, 70, 90-96.

Peselow, E. D., Robins, C., Block, P., Barouche, F. \& Fieve, R. R. (1990). Dysfunctional attitudes in depressed patients before and after clinical treatment and in normal control subjects. American Journal of Psychiatry 147, 439-444.

Power, M. J., Katz, R., McGuffin, P., Duggan, C. F., Lam, D. \& Beck, A. T. (1994). The Dysfunctional Attitude Scale (DAS): a comparison of forms A and $\mathrm{B}$ and proposals for a new subscaled version. Journal of Research in Personality, 28, 263-276.
Sahin, N. H. \& Sahin, N. (1992). How dysfunctional are the dysfunctional attitudes in another culture? British Journal of Medical Psychology, 65, 17-26.

Tavares, M. (1995). Escala de bem-estar - questionário demográfico. Laboratório de Psicoterapia e Psicodiagnóstico, Instituto de Psicologia - Unb.

Ward, C. H., Beck, A. T. \& Rascoe, E. (1961). Typical dreams. Archives of General Psychiatry, 5, 606-615.

Weissman, A. N. (1979). The Dysfunctional Attitude Scale: a validation study (Tese de Doutorado). Philadelphia: University of Pennsylvania.

Weissman, A. N. \& Beck, A. T. (1978). A development and validation of the Dysfuncitonal Attitude Scale: a preliminary investigation. Trabalho apresentado na Reunião Anual da Association for the Advancement of Behavior Therapy, Chicago.

Sobre os autores:

Mara Rúbia de Camargo Alves Orsini é mestre em Psicologia Clínica pela Universidade de Brasília - UnB (1998), professora efetiva de Psicopatologia Geral do Departamento de Psicologia da Universidade Católica de Goiás UCG; doutoranda pela Universidade de Brasilia, tendo como tema de pesquisa as relações entre características de personalidade e o Transtorno Depressivo Maior.

Marcelo Tavares é doutor em Psicologia Clínica pela United States International University (1993); coordena o Laboratório de Saúde Mental e Cultura do Instituto de Psicologia da UnB, onde desenvolve pesquisas em Avaliação Clínica, Psicoterapia Psicodinâmica, Intervenção em Crise e Prevenção do Suicídio. Na avaliação psicológica, focaliza a identificação precoce de risco, as técnicas projetivas e o psicodiagnóstico dinâmico e diferencial.

Bartholomeu Tôrres Tróccoli é mestre e doutor pela University of Wisconsin-Madison, professor do Departamento de Psicologia Social e do Trabalho da Universidade de Brasília-UnB, tem lecionado e prestado consultorias nas áreas de Avaliação de Programas, Análise de Dados de Pesquisas, Psicologia Social e Personalidade, Seleção de Pessoal e Psicometria. Suas atividades mais recentes incluem um novo curso sobre a Psicologia Evolutiva. 